\title{
Double-Layered $\mathrm{TiO}_{2}-\mathrm{SiO}_{2}$ Nanostructured Film with Self-Cleaning and Antireflective Properties
}

\author{
Xintong Zhang, Akira Fujishima*, Ming Jin, Alexei V. Emeline, and Taketoshi Murakami
}

Special Laboratory for Optical Sciences, Kanagawa Academy of Science and Technology, 3-2-1 Sakado, Takatsu-ku, Kawasaki-shi, Kanagawa 213-0012, Japan

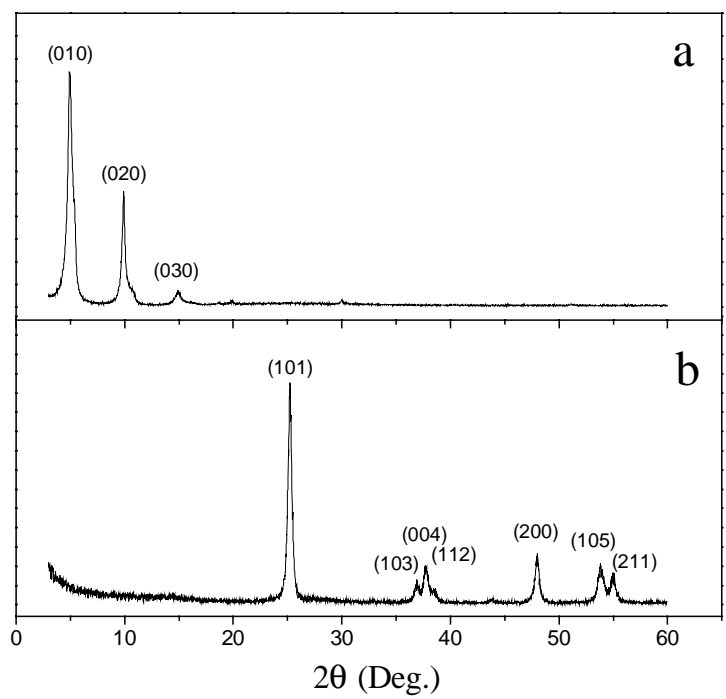

Figure S1: X-ray diffraction patterns of titanate nanosheet powders (a) and the powders calcined at $500{ }^{\circ} \mathrm{C}$ for 1 hour (b). This figure clearly showed that calcination caused the phase transition from lamellar structure of titanate nanosheet to anatase structure of $\mathrm{TiO}_{2}$. The X-ray diffraction patterns were recorded with a Rigaku RINT1500 diffractometer with graphite-monochromatized $\mathrm{Cu} \mathrm{K} \alpha$ radiation. The assignment of XRD pattern of nanosheet powders was based on the reported data of Sasaki's (J. Am. Chem. Soc. 1996, 118, 8329); while that of calcined powders was assigned according to the standard JCPDS card (\#78-2486). 